\title{
Data Security for Outsourced Cloud Datausing HASBE Scheme and RSA Algorithm
}

\author{
K.Venkatesh Sharma, B.Rakesh, D. Sasikala
}

\begin{abstract}
Cloud computing (CC) is the expertise over which every person is capable of sharing the reserves, amenities, and evidence amongst the individuals as a result of harnessing the internet in link. Subsequently, protection is a key concern on the facts pooled by means of the internet. In CC a numerous security issues prone to occur that includes confidentiality, integrity, authentication and/or thin well-honed edit control (TWHEC). In this research an innovative security replica has been aforethought. The design stipulates an approach across which secure communication besides data hiding from unauthorized punters can be got hold of. The security resolved with all categories of CC aspects similar to Platform as a Service (aaS), and Process aaS (PaaS), Software aaS, Storage aaS and Security aaS (SaaS), Network aaS (NaaS), Functions aaS (FaaS), Infrastructure aaS, Information aaS, and Integration aaS (IaaS), Database aaS (DBaaS), Application as a Service aaS and API aaS (AaaS), Management aaS (MaaS), Testing aaS (TaaS), etc., This anticipated system yield thin well-honed, mutable and ascendable statistics editing control by means of the manipulation of complex traits of Hierarchical (Ranked/Ordered) attribute-set-based encryption (HASBE). This multifarious qualities of blend of HASBE and Rivest-Shamir-Adleman (RSA) algorithm too. For instance, cyber individual healthiness record (IHR) aids sick persons to deal with his/her personal medicinal archives into a unified means that is to a great extent vital in storing, editing and partaking of the individual healthiness data. Further down encryption, it is impeding in accomplishing the TWHEC to CIHR data in an ascendable and effectual technique by using HASBE. Prevailing RSA encryption does not endow the data with high security in health. To fulfill ascendable, elastic, and thin well-honed edit control of subcontracted statistics in cloud. In this archetypal instigated a mish-mash RSA encryption with HASBE. Cyber IHR dispenses with patients to get along medical archives in a secure way, in which very significant concerns are the storing, access and distribution of individual wellness information. This composite process is responsible for three mode precautions, i.e. data precautions, certification and corroboration. In this report, the HASBE encryption algorithm has been put forward in realizing TWHEC to IHR data in an ascendable and effectual way.
\end{abstract}

Keywords :Thin Well-Honed Edit Control (TWHEC), Hierarchical (Ranked/Ordered) attribute-set-based encryption (HASBE), Rivest Shamir Adleman(RSA) algorithm, Individual Healthiness Record (IHR), Data security, Cloud computing (CC)

\section{INTRODUCTION}

CC ambiences standpoints its data or objects to be

Revised Manuscript Received on October31, 2019

* Correspondence Author

K.Venkatesh Sharma*, CSE Dept,CVR College of Engineering, Hyderabad, India. Email: venkatesh.cse@cvr.ac.in

B.Rakesh,,EnggDepartment, Al Musanna College of Technology

,Musanna, Oman. Email: betalarakesh@gmail.com

DSasikala, CSE Dept, CVR College of Engineering, Hyderabad, India..

Email: anjansasikala@gmail.com

imparted amongst servers, utilizes and personals, in records or information that are warehoused inside the cloud stay effortlessly affluent to procure to wholly. Owing towards this exposed user-friendly data accessing aspect, the personal files or data can be made use of by more utilizes in cloud that leads to invasive watch out of happening on data or files befallen to hazards additionally [6]. As soon as the meddlers procure $\log$ on to information, exploitation of it take control over a foremost imperil. The prowler may wipe out the innovative information; they cut short the transmission as well. Separately from the files and data cloud service providers smooth the progress of nit-picking applications whose security entails a bundle of awareness [7]. Unique shared predicaments crop up in the cloud stands that any distinct personal possibly will not retain the control done in the whereabouts of the data storing. It turns out to be essential for a cloud employer to make use of the reserve division and timetabling amenities afforded through the cloud check over benefactor in succession at the instance of dispensation it turns out to be vital to defend the statistics or archives of the beings. In the direction of surmounting this setback, haven at $\mathrm{CC}$ display place must stay instigated efficient. Ranges of security attributes have been investigated in this recommended CC model.

Hitherto, scholars have exhibited diverse haven replicas and procedures harnessed on them, then these replicas remained inept at resolving the entire categories of defense intimidations. An Individual Healthiness Record (IHR) is a budding fitness statistics expertise that persons be capable of editing, accomplishing and revealing their fitness- associated evidence [11], and that of others aimed at whom they stand authoritative, in a reserved, protected, and safe setting, subsequently owning an IHR know how to be a lifeguard.

For every single sick person, the IHR statistics ought to remain scrambled as a results of which, it is ascendable by the quantity of then existing utilizes retaining call up. Likewise, subsequently to hand are numerous holders (sick persons) in an IHR procedure and everyone would scramble their IHR archives with a dissimilar cluster of cryptographic key-ins, so that scalability, flexibility, and TWHEC of subcontracted statistics in $\mathrm{CC}$, in this exploration are accomplished, HASBE is propositioned by outspreading RSA [1],[12]. The anticipated system not only realizes elasticity entitled to its hierarchical edifice, also, after that again accede to litheness and TWHEC in stimulating multifarious traits of HASBE. Realistic method would remain towards scrambling the statistics prior to subcontracting. 


\section{Data Security for Outsourced Cloud Datausing HASBE Scheme and RSA Algorithm}

This one purports likelihood probes and intervallic corroboration to proctor the transformation of subcontracted facts by endowing an enhanced agenda. It condenses the load proceeding in the storing servers, even though is exposing the server's waywardness with an elevated probability. An ensuring tactic to contract out the IHR facts is, to encrypt these statistics in advance to subcontracting. At last, the IHR proprietor ought to resolve the resulting goings-on [11]: In what way to scramble their archives? and [2] which team of utilizes must stand permissible towards persuading log on to all portfolio? Every single scrambled portfolio editable to utilizes conferred with the consequent.Deciphers key-in. Above and beyond, about-turn is a new and significant trouble concerning this scheme. Aimed at this, the issue is vital towards revoking the edit rights after the utilizes deem that they are crucial [7].

\section{II.RELATED WORK}

In CC information is not put in storage in utilizer's computer, then ensue put into safekeeping in cloud storing that is instituted through unbiased observers. File enciphering and the deciphering procedures are created in RSA procedure where they retain anticipated RSA procedure for enciphering and the deciphering of smaller size files. The design encompassed distribution of individualistic files over encrypted IHR files towards setting aside TWHEC by lessening the complications of key-in managing. At this juncture, the editing strategies are imposed in utilizer's put $\mathrm{k}$ deficit overdue in steering active strategy amends. Representing an instance, if the sick persons do not aspire to scrutiny its facts via doctor immediately, squashing up his duty call, they can merely remove the cipher text manuscripts be consistent to the feature "doctor". This alteration is executed by other encipher methods wherever the accessible approach scarcity next to it. IHR services to one and all, even this can endure certain protection and confidentiality perils. It is vital to have TWHEC to guarantee concealment power across specific IHRs in cloud servers. Intact scheme makes certain to pass advanced built on health care protocols that include the Health Insurance Portability and Accountability Act (HIPAA) [8], but the cloud facility benefactors yet don't involve them. Basis after handling IHR facts to a private cloud [12] is an immense sick person's fitness statistics have been grasped involving a computer hard disk drive holding above four million unenciphered IHRs, embracing general details - Name, Date of Birth, age, email-id, address, etc., and specific details that includes medical record numbers, diagnoses and other statistics

\section{PROPOSITIONED MODEL}

In the anticipated system, the two methods that are executed are: RSA scheme and HASBE encryption. The drive of this effort is to extend the HASBE system to comprehend ascendable, elastic, and TWHEC in CC. This process seamlessly fits in a hierarchical (ranked/ ordered) edifice of system clients by relating to an allocation and to RSA algorithm. Security to data can be impacted by means of RSA algorithm [6], the high security of a data has been well confirmed by HASBE encipher.

The information proprietors aspire to preclude the server and unlawful utilizes finding the gist of their approachable portfolios retaining a concealment strategy. Precisely, this projected system is with the following trailing intents:

- TWHEC: Disparate utilizes can be approved to peruse diverse sets of files.

- Scalability: To sustain bulky and instable quantity of utilizes, the scheme must be tremendously ascendable, in stints of exertion in key-in managing, utilize managing, and working out and storing.

- Empower utilizes to make sure of the steadfastness of facts subcontracted.

- Facilitate user to make certain to prevail on the high security data on the cloud.

- RSA key: This key engendered from an RSA algorithm that is based on IHR data.

- HASBE key: This Key joins up RSA key with HASBE attributes

The intact technique smears to IHR that is cyber information on a person's fitness statistics. Cyber IHR provision [8-9] consents an individual to create, store, direct and share his personal health data in a centralized mode. At that time $\mathrm{CC}$ conferred limitless processing possessions and flexible storing. IHR facility benefactors budge the facts and submissions dropping their operating cost [5].

$\mathrm{CC}$ practice in notice entails five forms of groups: cloud facility benefactor, information proprietors, information customers, realm specialists, and reliable consultant. The cloud facility dealer governs a cloud to be responsible for the information storing facility.

1. Reliable Consultant Part

2. Realm Specialists Part

3. Information Proprietors Part

4. Information Customers Part

5. IHR Cloud Facility Benefactor Part.

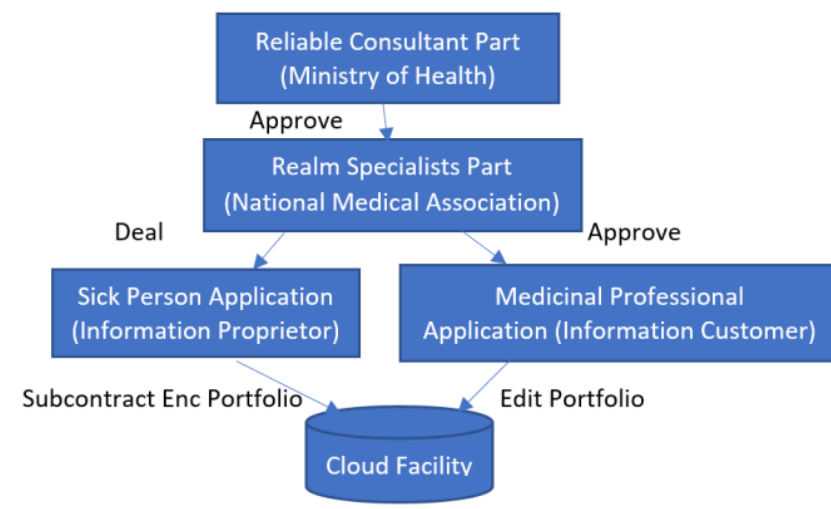

Fig. 1: HASBE Architecture

\section{Reliable Consultant Part}

The reliable consultant stays as the starting point otherwise parental consultant. It is in the authority of begetting and spreading out the scheme factors and origin master keys on top of commending the most senior realm establishments. Concerning this process, the Ministry of Healthcare $(\mathrm{MoH})$ is the unswerving expert witness.

The foremost roles of $\mathrm{MoH}$,

- Admin be able to login starting the home-based page and know how to transmit realm specialist registering.

Published By: 
- Towards setting up the process of engendering master pvt k- MK0 and a PK centered on worldwide successions of scheme aspects.

- Initiate master key for domain authority with public key (PK), master key MK0 and a collection of traits compatible to realm specialist.

\section{Realm Specialists Part}

Realm Specialist oversees dealing IHR proprietors and empowering statistics customers. In this process a solitary realm specialist so-called the National Medical Association (NMA) emanates beneath the $\mathrm{MoH}$.

$>$ NMA formerly enrolls into the reliable consultant. In the enrolment process the traits consistent to the realm specialist are precise. Likewise, an appeal aimed at realm interpretation is cast to reliable specialist in netting facilities. Barely, next to procure the realm key-in - PK and realm master key, the realm specialist can ratify utilizers in its realm.

$>$ Foremost roles of NMA:

- To confer PK for the sick persons to accomplish RSA algorithm.

- Registering and Interpretation of the specifics of medicinal authorities.

- Towards accord RSA secret (pvt) $\mathrm{k}$ for the medicinal authorities for deciphering the medicinal archives.

- Accomplish utilizer reversal.

\section{Information Proprietors Part}

Concerning this process sick persons are the information proprietors. Patent claim remains readily available that lets the sick person towards relating to IHR facility benefactor. Core roles of information proprietors' part:

- Sick persons formerly roll in this process and later register.

- Sick persons are able to position the edit dispensed by means of which persons are capable of interpretation of the portfolios and up and about put in enciphered portfolios towards the cloud.

- Sick persons claim accomplishes enciphering in dual levels. Foremost is the portfolio is scrambled with RSA key-in, i.e., is scrambled with sick persons revealed strategy and PK imparted through NMA. Next phase tallies, towards hypertext trait series constructed enciphering.

- Enciphered portfolio from the onset with enciphered RSA key-in remains up and about to put in towards the cloud.

\section{Information Customers Part.}

Medicinal experts are active as information customers. All over the medicinal specialized single-mindedness medics intermingle by the IHR facility benefactor.

- All hospice governors are logged in and initiates staffs by inputting their particulars. Registering minutiae analogously conferred towards NMA by means of netting facilities.

- Medicinal proficient claim realizes decipher of portfolios for all solitary member of staff by enquiring for equivalent pvt $\mathrm{k}$ constructed upon the qualities of the worker from NMA.

\section{IHR Cloud Facility Benefactors Part}

Liable intended for warehouse enciphered portfolios. It preprocesses the portfolio for provoking metadata for scrutinizing tenacity

\section{A. EXECUTION PRINCIPLE OF RSA ALGORITHM WITH HASBE}

The insinuated HASBE technique effortlessly enlarges the ASBE system to embrace the hierarchical edifice of system utilizes. Elicit that this augmented process replica entails an entrusted authorization, manifold domain establishments, and abundant users, alike the information vendors and patrons.

All publics of the conspiracy are apportioned a key structure that stipulates the aspects allied with the user's decryption key. Figure. 3 stands for HASBE Key data file structure of the cloud [3].

A realm sway is coupled with an exclusive identification (IDN) and a recursive property set $A=\{A 0, A 1, A 2 . \ldots$. .AM $\}$.Opt a sole IDN representing this information portfolio.

$>$ Impulsively decide on a symmetric information encipher key-in DEK $\leftarrow \mathrm{K}$, where $\mathrm{K}$ is the key-in space, and encipher the facts.

$>$ Delineate a tree edit assembly for the portfolio and encipher (IDN, RSA PK, and DEK) with using algorithm of HASBE that reinstates cipher text. to end with, the scrambled facts portfolio is amassed upon the cloud. Encrypt (IDN, PK, DEK). $M$ is the note to encipher

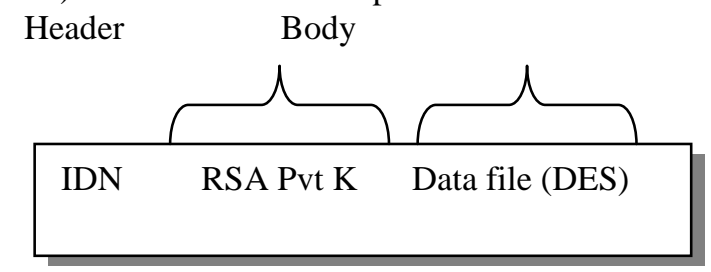

Fig. 2.Structure of facts portfolio atop of the cloud

Fabrication of Pvt K in Encryption

Step 1: Get hold of the IDN from data at Cloud server. Step 2: Engender a pvt k grouping IDN via Key Creator Object by RSA Algorithm.

Step 3: This pvt $\mathrm{k}$ is Key into the HASBE Process.

Step 4: In the HASBE endures DATA HEADER.

Step 5: The Data Header amalgam of IDN, Pvt K and DES KEY.

Step 6: Domain Authority Module breeds the data file key. Step 7: This key is unsystematically set off.

\section{Decryption:}

Decryption procedure is castoff to unravel the portfolios and the pvt $\mathrm{k}$ is bred to editportfolios from the cloud. In favor ofall end usersisolatedpvtksareengendered headed for earning $\log$ on toon or after any location with security. Decryption practices figured outin thereverse process. Step 1: Break open the Data Header with DES Key Step 2: Uncap the Pvt K over RSA Algorithm.

Step 3: Decrypt the data 


\section{Data Security for Outsourced Cloud Datausing HASBE Scheme and RSA Algorithm}

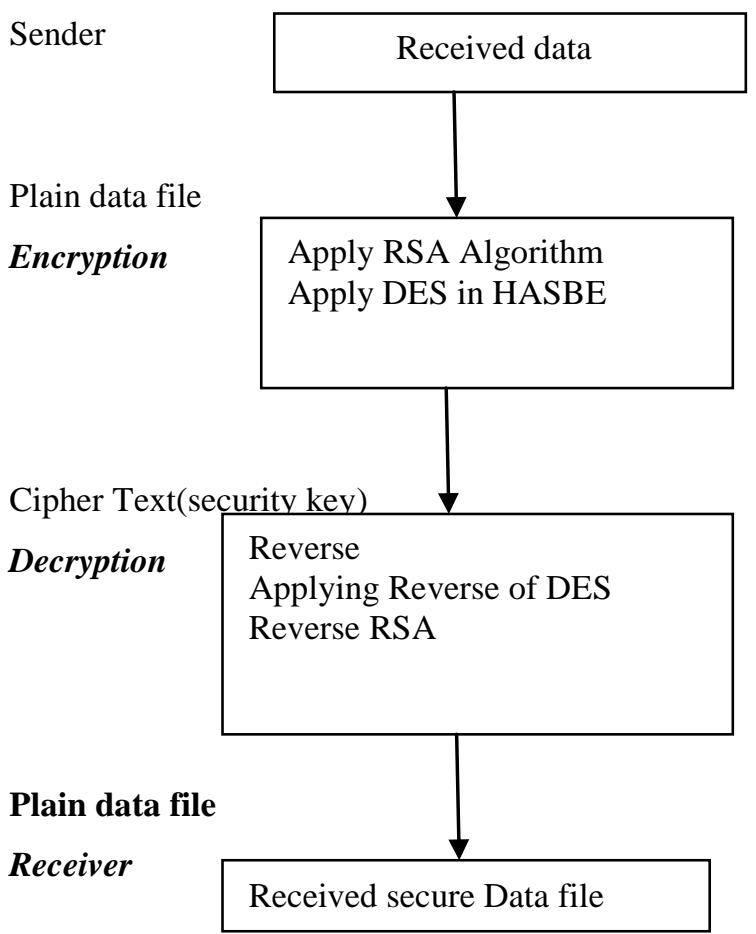

Fig 3.Overall structure of security design.

\section{CONCLUSION}

In CC status quo the IHR people are yet terrified of outsourcing their records owing to a series of security intentions. In this creative work, a latest HASBE header file has been purported that is a grouping of RSA Key and DES key, which is cast off for reliable distribution of IHRs in cloud server. HASBE has been employed to scramble the IHR data, to facilitate the sick persons who cannot permit editing only by individual domain manipulators, nevertheless manipulators from collective domains too with diverse proficient characters, memberships, and credentials. By means of this process, with the intention of instantaneously accomplishing: 1. TWHEC; 2. Expandability; 3. Data security in CC. This envisioned system furnishes better edit control than other attribute-based encryption (ABE) procedures. In the forthcoming opus, an improved depiction technique will be premeditated that can further lessen the vital management and policy intricacies

\section{ACKNOWLEDGMENT}

CVR College of Engineering Management, Principal, Computer Science and Engineering Department, HOD and staff members will always be my inspiration.

\section{REFERENCES}

1. Zhiguo Wan, Jun'e Liu, and Robert H. Deng, "HASBE: AHierarchical Attribute-Based Solution for Flexible and ScalableAccess Control in Cloud Computing", IEEE TRANSACTIONSON INFORMATION FORENSICS AND SECURITY, VOL. 7,NO. 2, APRIL 2012743.

2. Ming Li Member, IEEE, Shucheng Yu, KuiRen," Scalableand Secure Sharing of Personal Health Records in CloudComputing using Attribute-based Encryption", IEEETRANSACTIONS ON PARALLEL AND DISTRIBUTED SYSTEM,VOL. 11, 2012.

3. S.Jahid, P.Mittal, and N.Borisov, "Easier: Encryption-Based AccessControl in Social Networks with Efficient Revocation" Proc. of the6th ACM Symp. on Info., Computer and Comm. Security, pp. 411-415, 2011.

4. Michalewicz, Z.: Genetic Algorithms + Data Structures $=$ Evolution Programs. 3rd edn. Springer-Verlag, Berlin Heidelberg New York (1996).

5. C.O.Rolim, F.L.koch, C.B. Westphall, J.Werner, A.Fracalossi, andG.S.Salvador, "A Cloud Computing Solution For Patient's DataCollection In Health Care Institutions," proc. 2nd Int'l Conf. on ehealth,telemedicine and social medicine (ETELEMED '10), pp. 95-99, 2010.

6. B.Arun\& S,K. Prashanth, "Cloud Computing Security UsingSecret Sharing Algorithm" in Indian Journal of Research, ISSN-12250-1991, Volume:2|Issue: 3| March 2013.

7. S. Yu, C. Wang, K. Ren, and W. Lou, -Achieving secure, scalable, and fine-grained data access control in cloud computing, $\|$ in Proc. IEEE INFOCOM 2010, 2010, pp. 534-542.

8. G.Wang, Q. Liu, and J.Wu, - Hierachicalattibute-based encryption for fine-grained access control in cloud storage services, $\|$ in Proc. ACM Conf. Computer and Communications Security (ACM CCS), Chicago, IL, 2010.

9. Pasquale De Meo, Giovanni Quattrone, and DomenicoUrsinoIntegration of the HL7 Standard in a Multiagent System toSupport Personalized Access to e-Health Services. IEEEtransaction-Knowledge \& Data Engineering. Aug2011.

10. S. Yu, C. Wang, K. Ren, and W. Lou, -Achieving secure, scalable, and fine-grained data access control in cloud computing, $\|$ in Proc. IEEE INFOCOM 2010, 2010, pp. 534-542.

11. M.Li, S.Yu, Y.Zheng, K.Ren, and W. Lou, "Scalable and Secure Sharing of Personal Health Records in Cloud Computing UsingAttribute-Based Encryption," IEEE transactions on parallel anddistributed systems, vol. 24, no. 1, pp.131-143, January 2013.

12. Ravi Shankar Dhakar, Amit Kumar Gupta, "Modified RSA Encryption Algorithm (MREA)". Advanced Computing \& Communication Technologies (ACCT), Second International Conference, 2012.

13. Wang Cong, Wang Qian, RenKui, Cao Ning and Lou Wenjing ,"Toward Secure and Dependable Storage Services in CloudComputing," Services Computing, IEEE Transactions on , vol.5,no.2, pp.220-232, April-June 2012.

14. Hsiao-Ying Lin; Tzeng, W.-G.; , "A Secure Erasure Code-BasedCloud Storage System with Secure Data Forwarding," Parallel and Distributed Systems, IEEE Transactions on , vol.23, no.6, pp.995-1003, June 2012.

15. E. AbuKhousa, N. Mohamed, and J. Al-Jaroodi, "e-HealthCloud:Opportunities and Challenges," www.mdpi.com/journal/futureinternet, pp. 621-645, July 2012 